\title{
Changing funding patterns in tuberculosis
}

\author{
Stefan H E Kaufmann \& Shreemanta K Parida
}

Exactly 125 years after the discovery of the etiological agent of tuberculosis (TB), the disease remains as threatening as ever. The insufficient control measures in place today were developed in the late nineteenth and early twentieth centuries. A research and development gap in the latter half of the twentieth century has led to the lack of new approaches to controlling TB, and disease incidence is now escalating. With the increasing appearance of multidrugresistant TB, the emergence of HIV-AIDS and the threatening liaison between HIV and TB, new control measures are needed urgently. As the need for new TB drugs and vaccines has become more apparent, so too has the realization that new approaches are needed to funding and furthering TB research. Qualitatively, funding patterns for TB research have started to change from awarding small grants to individual investigators toward creating and funding large interdisciplinary consortia. Quantitatively, funding has increased from a few million dollars per year in the early 1980 s to 400 million dollars in 2005 , but needs to increase further. Translation of research and development to a product is cumbersome, and changes in funding are needed today to have $\mathrm{TB}$ control measures available in a decade at the earliest.

Imagine the following scenario: the discoverer of the number one killer of humans, a world-famous scientist of highest reputation, describes methods to diagnose the killer disease and claims to have developed a vaccine for its prevention and treatment. Because of the enormous impact of the disease and the high reputation of the scientist, the state government immediately supports a vast multicentric clinical trial. Unfortunately, the vaccine turns out to be a complete failure. This is a true

The authors are at the Max Planck Institute for Infection Biology, Department of Immunology, Charitéplatz 1, 10117 Berlin, Germany. e-mail: kaufmann@mpiib-berlin.mpg.de story of events that happened 115 years ago. The killer disease was tuberculosis (TB), and the scientist Robert Koch (1843-1910). Today, we have tools to diagnose $\mathrm{TB}$, we have drugs to treat the disease and we have a vaccine that prevents childhood $\mathrm{TB}$, at least partially. But all of this is the result of research performed between the end of the nineteenth and middle of the twentieth century, paid for at the time by taxpayers and by industry. Today, diagnosis of the disease is still done by staining of acid-fast bacilli in sputum - the same method developed 125 years ago by Robert Koch when he, for the first time, described the etiology of TB and the subsequent screening test by tuberculin developed eight years later ${ }^{1}$. In the early twentieth century, a vaccine for TB was developed by Albert Calmette (1863-1933) and Camille Guérin (1872-1961) at the Institute Pasteur, Lille, France which entered clinics in 1921 (ref. 2). It was termed bacillus CalmetteGuérin. The major drugs in current use for TB-streptomycin, para-aminosalicylic acid, isoniazid and pyrazinamide-were developed in the 1940s and 1950s (ref. 3).

We were falsely satisfied by these early achievements; they turned out to be insufficient. Despite an initial decline in TB incidence in the second half of the twentieth century, the incidence of the disease is now escalating. The main driving force is the HIV pandemic, which has allowed TB to reemerge so dramatically. This is further complicated by increasing incidences of multidrug-resistant (MDR) TB strains and, now, extensively drug resistant (XDR) TB strains that are virtually untreatable. Today, 9 million people are newly diagnosed with TB every year, of which more than 2 million die, with more than one third of these 2 million coinfected with $\mathrm{HIV}^{4}$. Fifteen million people are probably coinfected with HIV and TB; these individuals have more than 100-fold higher risk of developing disease within one year of infection than those infected with $M$. tuberculosis alone. MDR-TB strains account for 0.5 million cases of $\mathrm{TB}$, with the highest incidence in Eastern
European countries. Although in absolute numbers the incidence of XDR-TB is still low, it has been reported in 27 countries, and the virtual failure of all attempts to treat XDR-TB adds a new dimension to the threat in the context of the HIV-AIDS epidemic, as illustrated by a 98\% death rate of a recent outbreak in KwazuluNatal, South Africa ${ }^{5}$. It is no wonder that the World Health Organization (WHO) declared TB a global emergency as early as 1993 .

TB is a chronic disease. Hence, control failures do not become apparent within short time frames but surface only decades later. Thus, for example, the outbreak of MDR-TB in New York in 1990 costing $\$ 1$ billion was the result of the lack of sufficient control measures in operation much earlier ${ }^{6}$. What holds true for health care holds even truer for research. The present failure to adequately control TB is the result of lost opportunities in funding over the last few decades. Accordingly, increasing funding in this millennium will not produce a rapid return on investment until an interval phase of another decade or so has passed.

Here, we will briefly describe changing funding patterns over the last 25 years for TB research and development, and place this in the context of health care support and of funding for the other two members of the infamous trio of major killers: malaria and HIV (Table 1). We have chosen this time frame because AIDS entered public awareness in 1981 and soon became a driving force in revising our views on the global impact of infectious diseases, including TB. Between 1981, when HIV entered the stage, and 1993, when TB was declared a global emergency, funding was characterized by small-scale grants awarded to individual researchers. Since 1993, the funding situation has begun to change in response to the increasing awareness of the urgency with which new TB control measures are needed.

\section{Changing sources of funding}

In 1981, global TB-specific research was propelled for the most part by the US National 
a

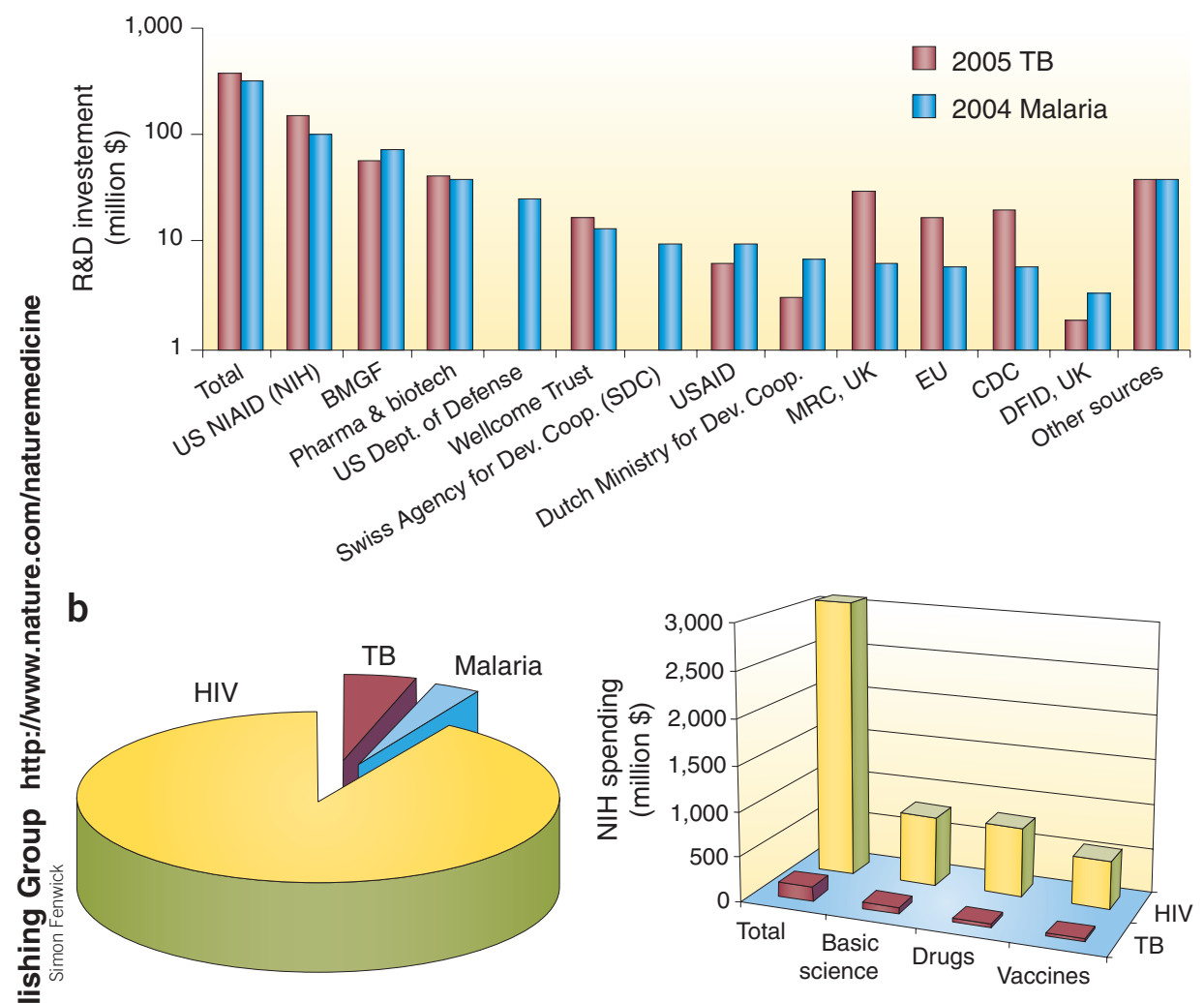

Figure 1 Funding situation for TB in comparison with malaria and HIV. (a) Research and development (R\&D) investment by largest funders for TB in 2005 compared with malaria funding in 2004. Sources: TB, ref. 13; malaria, Monitoring Financial Flows for Health Research 2005: Behind the Global Numbers, World Health Forum. (b) Pie diagram representing NIH funding for HIV (yellow), TB (violet) and malaria (purple); bar diagram representing spending on TB and HIV according to categories. USAID, US Agency for International Development; Dev. Coop., Development and Cooperation; EU, European Union; pharma, pharmaceutical companies; biotech, biotechnology companies; DFID, Department for International Development.

Institutes of Health (NIH) with small grants awarded to individual scientists. This situation slowly changed with the formation of small research groups in the late 1980s and early 1990s, who were funded for basic research with the aim of using results for developing interventions. These groups have taken on a new dimension this century, starting with the creation of TB research units in the developing world and TB vaccine and drug evaluation units by the US National Institute of Allergy and Infectious Disease (NIAID, under the NIH), the European Union's Fifth and Sixth Framework Programs (FP5 and FP6, respectively), the Wellcome Trust's Joint Infrastructure Funding program and, most recently, the Grand Challenges in Global Health program of the Bill \& Melinda Gates Foundation (BMGF). All of these are characterized by the assembly of larger consortia of several members from academic institutions and industry ${ }^{8-11}$.

According to NIH budget estimates, TB research expenses in the early 1980 s comprised a meager annual figure of \$1-2 million. Cases of TB in the United States increased by more than 20\% between 1985 and 1992, and this was reflected by the NIAID's increase in funding for TB research by tenfold: approximately \$3.5 million in 1991 to $\$ 35$ million in $1995^{7}$. The Institute's research program encompassed studies of the epidemiology and natural history of TB, as well as basic and applied research.

In 2000, the United Nations published the Millennium Goals, and in the following year the Global Fund for AIDS, TB and Malaria was founded. In 2006, \$190 million were earmarked for TB in 34 countries, and a total of $\$ 846$ million were approved by the Global Fund for TB during the last 6 rounds of funding. This is only $15 \%$ of the total expenditure of the Global Fund, less than that for malaria (27\%) and HIV-AIDS (57\%). Virtually all of this funding was for TB control programs, with very little dedicated to research, most of which was purely operational ${ }^{12}$. The BMGF, which was founded in 2000 , became an additional driver of TB research and is having increasing impact. The BMGF has the astonishing sum of $\$ 30$ billion, a sum that will double over the next years after Warren Buffet's historic gift last year. The BMGF earmarked nearly $\$ 60$ million in 2005 for TB-related research, including $\$ 10.3$ million for operational research. More recently, new commitments were made in terms of the TB drug accelerator program (with an additional \$40 million promised for 2007 and 2008) and \$104 million for the Global Alliance for TB Drug Development for the period 2006-2010. Thus, we have come a long way in terms of total amounts spent annually on TB research: from a few million dollars in 1981 , to $\$ 50$ million in the period 1993-1994, to $\$ 400$ million in 2005.

Recently, the Treatment Action Group (TAG) has made publicly available more precise information about the current funding situation in TB research and development ${ }^{13}$. The $\$ 400$ million invested in TB research in 2005 derived from more than 40 donors. Of these, $69 \%$ were from the public sector, $20 \%$ from foundations and $11 \%$ from industry. Some contributions may be underestimated because of nondisclosure. The top funding supporters were the NIH (US), BMGF (US), Medical Research Council (MRC, UK), Centers for Disease Control and Prevention (CDC, US), Wellcome Trust (UK) and European Union, with \$158 million, \$57 million, \$31 million, \$20 million, \$18 million and \$18 million, respectively (Fig. 1a). Support for basic science, infrastructure development and operational research to pave the way for validation of new tools for TB control amounted to $\$ 188$. Basic research on TB amounted to $\$ 94$ million (24\% of total); applied or unspecified research received \$4 million (11\%) and operational research received $\$ 50$ million (13\%). Two hundred and six million dollars were spent on new tools for TB control: $31 \%$ (\$120 million) on development of drugs, $18 \%$ (\$70 million) on vaccines and $4 \%$ ( $\$ 16$ million) on diagnostics. By far the largest share came from the US government, with a laudable $47 \%$. However, to put this in perspective, the $\$ 158$ million spent by the NIH on TB research (NIAID alone provided $76 \%$ of this funding) represents slightly more than $5 \%$ of the $\$ 2,921$ million that the NIH spent on HIV-AIDS research (Fig. 1b); and with $\$ 187$ and $\$ 183$ million, NIH funding both for smallpox and for anthrax, respectively, exceeded that for TB research.

Extensive efforts have been made to estimate the amount of investment in improved health care and accelerated basic research needed to reverse global incidences of TB. The Stop TB Millennium Plan 2006-2015 has calculated a total additional research and development 
need of $\$ 9$ billion over the next ten years, split into \$59 million, \$418 million and \$209 million per annum for new diagnostics, drugs and vaccines, respectively. Based on the current trend of investment in new tools, funding must rise nearly fivefold, from $\$ 206$ million to $\$ 1,050$ million per annum, to achieve the targets of the Global Plan.

\section{Pushing and pulling TB research}

TB research is fascinating. It is multidisciplinary, comprising the whole spectrum of biomedical research: molecular genetics of host and pathogen, cell biology, immunology, structural biology, clinical research, biochemistry and of course all the '-omics'. Yet TB research is also arduous, being painstakingly time-consuming and requiring the highest safety levels. The motivation for academic scientists to perform research on any host and pathogen crosstalk is influenced by many considerations. Basic research primarily aims at increasing knowledge, ideally of general relevance. Rarely will TB be selected for a basic model to provide generalizable insights. Rather, the lure of TB research lies in targeting highly sophisticated basic research to meet specific societal needs. Recognition of this distinction is important when considering funding strategies. Before the mid-1990s, much of the funding was aimed at supporting small-scale basic research in TB, with a vague and unfocused expectation that the findings might one day lead to better treatment or prevention options. One of the first funding programs earmarked for TB was the Immunology of TB (IMMTUB) Program of WHO in 1983, which provided small amounts of money to support basic research, typically

excluding any large-scale studies. Although the output of results per dollar spent in this phase was remarkable, such studies primarily served to identify basic mechanisms in TB pathogenesis and immunology, and did not lead to better control strategies. The program did, however, help to raise awareness of the need for research in TB and laid the groundwork for many later studies including development of new tools.

In contrast to academic TB research, research and development in industry is mostly oriented toward profit-making - to ultimately develop a product that can be sold for a price that will recoup the costs of development and allow for a certain amount of profit. Here again, TB research is not highly attractive because prices need to be low for a disease that prevails in developing countries. Accordingly, industry efforts in TB have been meager, with only 3 of the 1,393 drugs that were approved between 1975 and 1999 by the US Food and Drug Administration and the European Agency for the Evaluation of Medicinal Products being for

Table 1 Disease burden and funding comparison 2001-2002

\begin{tabular}{lllllll}
\hline Condition & $\begin{array}{l}\text { Global } \\
\text { disease burden } \\
\text { (million DALYs) }\end{array}$ & $\begin{array}{l}\text { Global } \\
\text { disease } \\
\text { burden (\%) }\end{array}$ & $\begin{array}{l}\text { Death } \\
\text { (millions) }\end{array}$ & $\begin{array}{l}\text { Global } \\
\text { disease } \\
\text { deaths (\%) }\end{array}$ & $\begin{array}{l}\text { R\&D } \\
\text { funding } \\
\text { (million \$) }\end{array}$ & $\begin{array}{l}\text { R\&D } \\
\text { funding } \\
\text { per DALY }\end{array}$ \\
\hline HIV, AIDS & 84.458 & 5.7 & 2.777 & 4.9 & 2,049 & 24.26 \\
\hline Malaria & 46.486 & 3.1 & 1.272 & 2.2 & 288 & 6.20 \\
\hline TB & 34.736 & 2.3 & 1.566 & 2.7 & 378 & 10.88 \\
\hline
\end{tabular}

Source: Ch. 5, Monitoring Financial Flows for Health Research 2005: Behind the Global Numbers, World Health Forum. DALYs, disability adjusted life years lost due to disease and injury, a measure of healthy life lost; $R \& D$, research and development.

the treatment of TB (Table 2$)^{14}$. By comparison, 179 drugs were licensed for cardiovascular diseases and 89 for noninfectious respiratory diseases. The outlook in the vaccine field is even more dismal; only in recent years have new vaccine candidates entered phase 1 clinical trials.

Obviously, the incentives for both academic researchers and industry were by themselves insufficient for pushing TB research forward. By the mid-1990s, funding slowly shifted toward multidisciplinary research strategies. The most significant changes have been the creation of large consortia bringing together academic and industrial expertise. The European Union FP6 and, in part, its predecessor, the FP5, have funded major TB consortia. In total, the FP6 has spent a total of $\$ 600$ million on poverty-related diseases with $\$ 275$ million (46\%) allocated to basic and translational research. Approximately $24 \%$ of the funding for basic and translational research went to TB (approximately $\$ 143$ million), $51 \%$ to HIV and 25\% to malaria. The European Union integrated projects have defined targets, such as the development of drugs or vaccines for TB, focused on preclinical research but also including phase 1 clinical trials. Yet they have also succeeded in integrating some basic research and industrial development to keep the pipeline filled.

The BMGF Grand Challenges have advanced this concept even further. In 2002, the BMGF asked the scientific community from all over the world to name major roadblocks toward the largest health problems, which are unequally distributed in developing and industrialized countries. In 2003, the BMGF-on the basis of the input from the scientific community-defined a number of Grand Challenges in Global Health ${ }^{15}$. In the field of TB, this has led to the formation of three multidisciplinary and multinational consortia. These Grand Challenges try to bring together researchers of different expertise and nationalities to find innovative solutions and also provide strategies to make these solutions accessible and free of patent-related costs to those who need them most. Attempts are being made to more frequently bridge gaps between wet labs and field sites. For example, the Grand Challenge project 'Biomarkers of protective immunity against $\mathrm{TB}$ in the context of HIV/AIDS in Africa' tries to combine highly sophisticated immunologic analyses and global gene expression profiling developed in laboratories of the industrialized world with clinical studies at different field sites in Africa ${ }^{16}$.

Another strategy for furthering TB research is to explicitly support public-private partnerships, partnerships that bridge the gap between basic academic research and development and the testing of research products. The NIAID has funded public-private partnerships in TB. New programs such as the Small Business Innovation Research program (2.5\% of the agency's extramural budget) and the Small Business Technology Transfer Research Program encourage research and development on products with the potential for commercialization. Similar efforts have been made by the

Table 2 New chemical entities (NCEs) approved (1975-1999) by therapeutic area, disease burden and sales ${ }^{14}$

\begin{tabular}{lllll}
\hline Therapeutic area & $\begin{array}{l}\text { Approved NCEs } \\
\text { (number) }\end{array}$ & $\begin{array}{l}\text { Approved NCEs } \\
\text { (\% of total) }\end{array}$ & $\begin{array}{l}\text { NCEs per million } \\
\text { DALYs }\end{array}$ & $\begin{array}{l}\text { Drug sales (million } \\
\text { \$ per DALY) }\end{array}$ \\
\hline Total & 1,393 & 100 & 1.01 & 148 \\
\hline HIV, AIDS & 26 & 1.9 & 0.37 & 44 \\
\hline Malaria & 4 & 0.3 & 0.10 & 5 \\
\hline TB & 3 & 0.2 & 0.11 & 11 \\
\hline $\begin{array}{l}\text { Respiratory } \\
\text { (noninfectious) }\end{array}$ & 89 & 6.4 & 1.44 & 307 \\
\hline Cardiovascular & 179 & 12.8 & 1.25 & 283
\end{tabular}




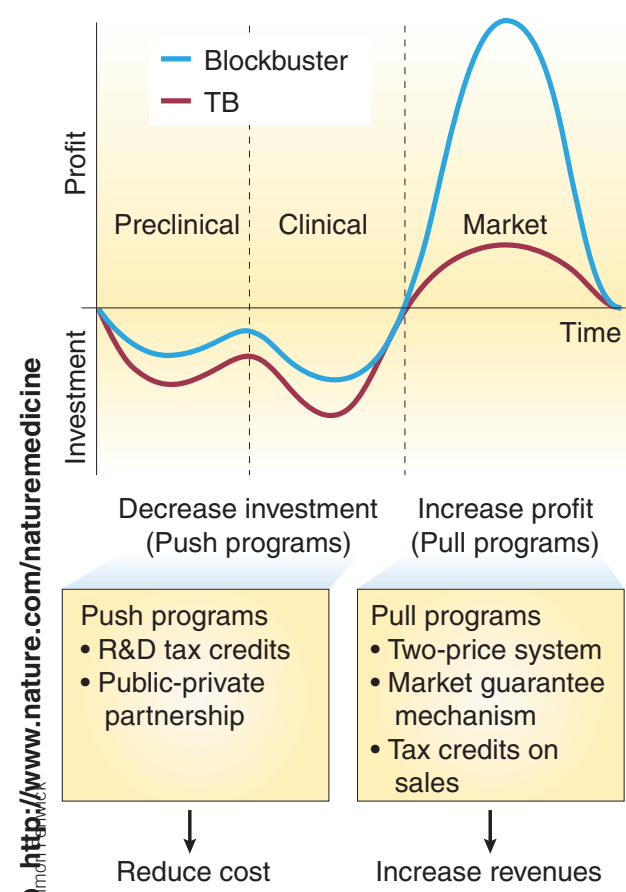

Figure 2 Duality of TB vaccine and drug development. Costs of investment for TB vaccine development (preclinical and clinical) are high owing to many requirements. Various 'push' mechanisms to decrease the costs in synergy with 'pull' mechanism to increase revenue with the successful introduction of the vaccine or drug are critical.

Wellcome Trust through new programs such as the Biomedical Science Funding Program and the Technology Transfer Program, supporting trials relating to health problems of significance in developing countries and facilitating proof-of-concept or safety studies of new technology requiring regulatory approval. The German Government has founded 'Vakzine Projekt Management'17 in order to bring vaccines, including a TB vaccine, to the proof-ofconcept stage so that they become attractive for industry.

Foundations have also seen the need for more strategic research and development of aspects not covered by industry. The major public-private partnerships (PPPs) founded to discover and develop new, affordable TB diagnostics, vaccines and drugs are the AERAS Global TB Vaccine Foundation (previously Sequella Foundation) for vaccines; the Global Alliance for TB Drug Development (TB Alliance) for drugs; the Foundation for Innovative New Diagnostics (FIND) for diagnostics; and the Consortium to Respond Effectively to the AIDS-TB Epidemic (CREATE), supported in large part by the BMGF, for the development of new types of public health intervention to reduce HIV-related $\mathrm{TB}^{18,19}$. A number of other foundations and national governments also support these PPPs to varying degrees. In addition, the TB Alliance has been supported by funding from the Dutch government as well as the Rockefeller Foundation.

Also important has been the realization that comparative large-scale test series need to be performed to assess the efficacy of new vaccine candidates and new drugs. To this end, the NIAID has established the TB Vaccine Testing Contract at Colorado State University, Fort Collins, Colorado, USA, which also provides mycobacterial research reagents to investigators globally free of charge; and the Tuberculosis Anti-microbial Acquisition and Coordinating Facility at Birmingham, Alabama, USA to facilitate the discovery of novel vaccines and drugs $^{20-23}$. The Tuberculosis Animal Research and Gene Evaluation Taskforce (TARGET) was formed at John Hopkins University in response to a request from the NIAID in 2002 to enable the modeling of human TB in multiple animal species using defined protocols and the testing of defined mutants of $M$. tuberculosis ${ }^{24}$. These facilities provide vaccine, drug and mutant testing for the TB scientific community all over the globe.

Industry seems also to realize the need for academic research activities. The large pharmaceutical company Novartis has established the Novartis Institute for Tropical Diseases in Singapore, which focuses on TB drug research as a major topic, and has just established a Vaccine Institute in Siena, Italy. AstraZeneca has made similar efforts in Bangalore, India, focused on TB drug discovery, and GlaxoSmithKline has a facility in Tres Cantos, Spain devoted to research on drugs for neglected diseases, including TB and malaria.

Finally, and of increasing importance as drugs and vaccines start entering clinical trials, is the establishment of well equipped and experienced field sites. This needs enormous efforts in terms of capacity-building in regions where $\mathrm{TB}$ is highly prevalent, such as Africa, Southeast Asia and Eastern Europe. The European Developing Countries Clinical Trials Partnership funded by the European Union, for example, has recently embarked on a journey toward this goal in Africa ${ }^{25}$. Time is too short to judge whether this courageous goal will be reached and will bring new momentum into the development of top research centers in the developing world. The NIH, CDC and several US universities have established many field units and research centers in the developing world to address the health issues of the host country while also aiming at local capacitybuilding, as well as infrastructure development for future large-scale phase 3 clinical trials. Although it may be more attractive for researchers and funding organizations to select sites in developing countries that have already reached a certain stage of maturity, in the long term this may create another two-class system and create a few overcrowded centers for clinical research and trials in 'a sea of nothingness. Clearly this is not to be recommended. Capacity-building in developing countries should consider the creation of well equipped units for top researchers in close association with, but largely independent from, universities and other clinical centers. A rush strategy would be shortsighted; only long-term investment will pay off. It is indeed gratifying to see that clinical centers in Africa receive increasing support for collaborative work from international funding organizations, but frustrating that at the same time these centers are drained of adequate medical care and research capacities by agencies that entice doctors, technicians, nurses and health care workers to accept more attractive positions in richer countries. Although such concerns may reach beyond direct goals of funding organizations, a future awareness of these problems needs to be integrated into future strategies. This could include fellowships for talented young researchers from developing countries to return to their home countries with sufficient funding to establish own laboratories and research groups.

To bring forward new vaccine and drug candidates for TB through the private sector or public-private partnerships, additional incentives may be needed. Classical push programs, which judge and fund research on the basis of proposed work plans, may be insufficient. Pull programs whereby funding agencies promise a secure market by guaranteeing advanced purchase of a number of products for a fixed price, may be needed in addition. Such a strategy has been successfully used as an incentive for the introduction of a meningococcal vaccine in Britain in late 1999, dropping the incidence of group C meningococcal disease by $90 \%{ }^{26}$. Further incentives, such as tax reduction for the industry partner earmarked for specific developments such as TB vaccines and drugs, and a tiered price system, whereby clients in developing countries pay less than those in industrialized countries, could be used as an additional support mechanism. Innovative strategies will be needed, and it is rewarding to see that funding organizations appear ready to exploit these as flexibly as possible (Fig. 2).

Incentives may be particularly needed for vaccines. First, in clinical phase 3 trials, any new vaccine candidate will have to be compared to BCG; second, a clinical trial will be of long duration and will require large cohorts. Yet BIO Ventures for Global Health recently calculated that even a new TB vaccine may find 
a market, with a global annual market peak for a BCG replacement vaccine of $\$ 450$ million, for a booster vaccine on top of BCG of nearly $\$ 800$ million and for a new prime-boost strategy of approximately $\$ 1$ billion ${ }^{27}$. Whether this is sufficiently attractive for big pharmaceutical companies to more actively engage in this endeavor remains to be seen.

\section{Conclusions}

Funding for TB research has changed markedly, both in quantitative and qualitative terms. In quantitative terms, during the last 25 years, an impressive increase of almost 200 -fold has occurred. In qualitative terms, funding has moved from small projects to multidisciplinary consortia in an attempt to bring together public and private activities, as well as highly sophisticated research labs with clinical field sites. Perhaps this may affect the depth of research that can be performed. Yet such an interdisciplinary and translational strategy is needed for the development of new control measures for TB.

Funding for TB research could develop a new paradigm by integrating innovative cutting-edge research with strategic and developmental activities to come up with a product that is accessible where it is most needed, with a focus on a limited number of the most critical roadblocks. Pasteur described this combination about 150 years ago: "No science can be called an applied science. There is science and the application of science which are bound together like the fruit to the tree." This message has been forgotten and needs to be revitalized.

The global threat of TB increases. To reverse this trend we need increased funding. First, we need to promote available control measures such as Directly Observable TreatmentShort-Course (DOTS) and DOTS Plus for treatment of TB and Expanded Program on Immunization for newborn BCG vaccination. In addition to these short-term goals, we need new products to replace current and insufficient diagnostic, treatment and preven- tion measures developed between the end of the nineteenth and the middle of the twentieth century. The sputum smear positive is insufficient for diagnosis of TB in approximately half of all cases, including most HIVM. tuberculosis coinfected individuals. Treatment of MDR-TB with second-line drugs easily increases costs by a factor of 100 or more and is of little value for the treatment of XDR-TB. Compelling evidence from clinical trials tells us that BCG has no impact on adult pulmonary TB. Hence, the full armamentarium for infectious disease control is needed for TB: more specific and more sensitive diagnostics, ideally with prognostic value; better drugs, notably those that reduce the time of treatment from several months to weeks (for example, by attacking dormant tubercle bacilli); and a vaccine that not only affords protection for toddlers but also for their parents. These measures are not likely to become available before the next decade, and then only if funding is increased today. The choice of the right funding strategies, which promote the combination of different expertises of academic institutions, clinics and industry, are of critical importance. Money will remain restricted, and therefore we have to focus to get the best possible results out of the limited resources available. The cost of TB for the world has been calculated as high as $\$ 56$ billion. The estimated cost for TB control measures is calculated as $\$ 47$ billion, with $\$ 9$ million for research and development of new diagnostics, drugs and vaccines. It is an enormous amount of money, yet in 10 to 20 years' time, the world may be grateful that we have changed directions toward funding today to save an estimated 35 million lives by 2015 .

\section{ACKNOWLEDGMENTS}

The authors are thankful to M.L. Grossman for editorial help and to A. Ginsberg, C. Sizemore, P.-H. Lambert, O. Olesen and H. Laang for suggestions on the manuscript.

\section{COMPETING INTERESTS STATEMENT}

The authors declare that they have no competing financial interests.
1. Koch, R. Die Aetiologie der Tuberculose. Berliner klin. Wochenschr. 19, 221-230 (1882).

2. Fine, P.E. Rev. Infect. Dis. 11 (suppl. 2), S353-\$359 (1989).

3. Zhang, Y. Annu. Rev. Pharmacol. Toxicol. 45, 529-564 (2005).

4. World Health Organization. <http://www.who.int/tb/ publications/global_report/2006/pdf/full_report_correctedversion.pdf> (2006).

5. Singh, J.A., Upshur, R. \& Padayatchi, N. PLoS Med. 4, e50 (2007).

6. Frieden, T.R., Fujiwara, P.I., Washko, R.M. \& Hamburg, M.A. N. Engl. J. Med. 333, 229-233 (1995).

7. US National Institute of Allergy and Infectious Diseases. <http://www3.niaid.nih.gov/news/newsreleases/1996/tbtip.htm> (1996)

8. National Institute of Allergy and Infectious Diseases. <http://www3.niaid.nih.gov/researchfunding/> (2007).

9. European Commission. Research. <http://ec.europa. eu/research/health/poverty-diseases/projects/104_ en.htm> (2007).

10. TBVAC.ORG <http://www.tb-vac.org/> (2007).

11. Bill \& Melinda Gates Foundation. <www.gatesfoundation. org $>$ (2007).

12. The Global Fund to Fight AIDS, Tuberculosis and Malaria. <http://www.theglobalfund.org/en/apply/ current/> (2006).

13. Feuer, C. Tuberculosis Research and Development: A Critical Analysis (Treatment Action Group, New York, 2006).

14. Trouiller, P. et al. Lancet 359, 2188-2194 (2002).

15. Grand Challenges in Global Health. <http://www.gcgh. org > (2007).

16. Max Planck Institute for Infection Biology. <http://www. biomarkers-for-tb.net/> (2007).

17. Vakzine Projekt Management $\mathrm{GmbH}<$ http://www.vak zine-manager.de/> (2007).

18. Colorado State University, College of Veterinary Medical \& Biomedical Sciences, Microbiology. <http:// www.cvmbs.colostate.edu/microbiology/tb/top.htm> (2007).

19. Tuberculosis Antimicrobial Acquisition and Coordinating Facility. <http://www.taacf.org/> (2007).

20. AERAS Global TB Vaccine Foundation. <http://www. aeras.org/> (2007).

21. TB Alliance. Global Alliance for TB Drug Development <http://new.tballiance.org/> (2007).

22. Foundation for Innovative New Diagnostics. <http:// www.finddiagnostics.org/> (2007).

23. Consortium to Respond Effectively to the AIDS/TB Epidemic. <http://www.tbhiv-create.org/> (2007).

24. Tuberculosis Animal Research and Gene Evaluation Taskforce. <http://webhost.nts.jhu.edu/target/> (2007).

25. European \& Developing Countries Clinical Trials Partnership. <http://www.edctp.org/> (2007).

26. Kramer, M. \& Glennerster, R. Strong Medicine: Creating Incentives for Pharmaceutical Research on Neglected Diseases (Princeton University Press, Princeton, New Jersey, USA, 2004).

27. BIO Ventures for Global Health. Washington, DC $<$ http:// www.bvgh.org/documents/BVGHTBVaccineReport106FINAL.pdf (2006). 